\title{
Antimicrobial Assessment of a Novel Humectant-Hand-Sanitizer against Microbes Transferred To Human Palms from Mobile Phones With/Without Flip Covers
}

\author{
Majumder $\mathrm{S}^{1,2 *}$, Mazumdar $\mathrm{S}^{3}$, Pyne $\mathrm{A}^{2}$, Das $\mathrm{A}^{2}$, Sadhukhan $\mathrm{A}^{2}$ and Mitra \\ $\mathrm{AK}^{2}$ \\ ${ }^{1}$ Department of Food Science and Agricultural Chemistry, McGill University, Canada \\ ${ }^{2}$ Department of Microbiology, St. Xavier's College, Kolkata, India \\ ${ }^{3}$ School of Life Sciences, Manipal Academy of Higher Education, India
}

*Corresponding author: Satwik Majumder, Department of Food Science and Agricultural Chemistry, Macdonald Campus, McGill University, 21,111 Lakeshore, Ste Anne de Bellevue, Quebec H9X 3V9, Canada, Tel: +14385304088; Email: satwik.majumder@mail.mcgill.ca

\section{Abstract}

The lack of proper sanitation and hygiene over the years has contributed to a rapid increase in communicable diseases, especially in developing nations like India. This problem warrants investigation of the potential routes responsible for transmitting microbes and infections to an individual. In this study, we have assessed bacterial and fungal load in covered (with flip covers) and uncovered (without flip covers) mobile phones, and the potential transfer of these microbes from phone surfaces to human palm surfaces. The bacterial load (in cfu) in mobile phones with flip covers was 13-fold higher than that of the uncovered phones meanwhile, the fungal load was relatively higher in the uncovered phones. The transfer of bacterial population (in $\mathrm{cfu}$ ) from the covered mobile phones to the palm surfaces was 14.5 -fold higher than that of the uncovered phones. Similarly, fungal transfer was relatively higher from the uncovered phones to the palm surfaces. We further developed a novel alcohol-based Humectant-Hand-Sanitizer (HH-sanitizer) and its sanitization efficiency (in \%) was compared with one of the most popular Hygiene products in India, Dettol, against microbes obtained from human palm surfaces (before and after rubbing over mobile phones), and a group of opportunistic pathogens: Enterobacter aerogenes, Escherichia coli, Klebsiella pneumoniae, Pseudomonas aeruginosa, Staphylococcus aureus, Enterococcus sp., Bacillus cereus, Listeria monocytogenes, Candida albicans, and Aspergillus sp. The sanitization efficiency (SE \%) of the HH-sanitizer on palm surfaces was $98.78 \pm 1.82 \%$ and $91.27 \pm 4.24 \%$ against bacterial and fungal isolates respectively, whereas the SE \% for the Dettol sanitizer was $94.62 \%$ against bacteria and $45.89 \%$ against fungi. In vitro antimicrobial assessment against the tested organisms showed the minimum inhibitory concentration of the HH-sanitizer to be 8-fold lower than the Dettol sanitizer. Thus, the HH-sanitizer has the potentiality to be an alternative option. Since bacterial adherence to mobile phones with flip covers and their transfer to human hands was significantly higher and as the common infections, we are prone to are caused more by bacteria than fungus, either way, avoidance of flip covers in phones/laptops/tablets, etc. is recommended to avoid transmittable infections and achieve better hygiene.

Keywords: Public Health and Hygiene; Communicable/Transmittable Disease; Mobile Phones; Human Palm Surfaces; Microbial Adherence; Opportunistic Pathogens; Sanitization Efficiency; Humectant-Hand-Sanitizer; Dettol Hand Sanitizer 
Abbreviations: SE: Sanitization Efficiency; HH-Sanitizer: Humectant-Hand-Sanitizer; CFU: Colony Forming Units.

\section{Introduction}

According to the World Health Organization (WHO), infections associated with microorganisms cause death to over 17 million people worldwide every year [1]. Around $80 \%$ of these microorganisms are transferred from the non-sanitized human hands to food, objects, or people thus contributing to communicable diseases [2]. Public sanitation and hand hygiene are universally recognized by the Centers for Disease Control and Prevention, the World Health Organization, and many other health experts as one of the most effective phenomena in disease prevention [3]. This rising threat especially in developing nations and highly populated countries like India warrants investigation of the potential routes responsible for microbial transmission to human hands.

Microbial adherence is a prerequisite for the initiation of diseases [4]. Recently, a study revealed that mobile phones, without which we nowadays cannot sustain ourselves contain a ten times higher microbial population than toilet seats [5]. Mobile phones were also reported to contribute to nosocomial infections due to the wide phone use in hospitals by the patients, visitors, health care staff, etc. without adequate sanitation [6]. In addition to that, mobile phones of patients and hospital staff have been also reported to harbor $40 \%$ and $20 \%$ of pathogenic bacteria, respectively [6]. Owing to the moisture, optimum temperature of the human body especially our palms, and the heat generated by mobile phones contributes to harboring microbes at alarming levels. When we consider a phone's daily contact with the face, mouth, ears, and hands, the dire health risks of using germinfested mobile devices are obvious.

As stated by Jain et al., microbial habitation on hands could be divided into resident and transient floras [7]. For instance, Staphylococcus aureus, Staphylococcus epidermidis, and Enterococcus faecalis colonize the deeper skin layers and are resistant to mechanical removal, whereas, Escherichia coli and Pseudomonas aeruginosa colonize the superficial layers of skin in a short period of time [7]. To eliminate such opportunistic pathogens and improve hygiene, alcohol-based hand rubs or sanitizers are widely endorsed [8]. Studies have shown that handwashing often removes the fatty acids from the skin resulting in cracked skin that provides an entry portal for pathogens whereas, the addition of emollients in hand sanitizers overcomes this limitation $[9,10]$. Moreover, the application of alcohol-based hand sanitizers is also reported to be a contributing factor in reducing gastrointestinal illnesses, respiratory tract infections, skin infection, etc in households, schools, laboratories, healthcare settings, etc. [7,11,12]. There are many hand sanitizers in the market with varying degrees of effectiveness registered in the National Agency for Food and Drugs Administration and Control. Despite the label claim of $99.9 \%$ effectiveness against harmful germs by several of these sanitizers, researchers have observed these products to fail significantly in reducing the microbial load from human hands $[7,13,14]$. Therefore, there is an urgent need for a novel strategy to develop an alcohol-based hand sanitizer that would not only be effective against a wide range of pathogens but also be honest to the public.

Several studies have been conducted to assess microbial load on mobile phones however, to the best of our knowledge there has been a knowledge gap on the potential transfer of microbes from mobile phones with and without flip covers to human palm surfaces. Thus, the present study quantifies the microbial population in covered (with flip covers) and uncovered (without flip covers) mobile phones and assesses their potential transfer to human palm surfaces. The research also involves the development of a novel Humectant-HandSanitizer. Its antimicrobial efficiency was compared with a commercially available product, Dettol Hand Sanitizer, against microbial population on human palm surfaces, and a group of opportunistic pathogens. The work generated enough evidence to recommend avoidance of flip covers in electronic devices such as phones/laptops/tablets etc. as they could be a potential source of microbial transfer to human hands. The study also suggested the use of Humectant-Hand-Sanitizer as an alternative to other commercially available hygiene products. The study focuses on the Indian microbiome, and the results could be extrapolated to the South-East Asian population with similar geographical conditions.

\section{Materials and Methods}

\section{Sample Collection}

Six mobile phones were collected from six volunteers in Kolkata, India. Three out of the six mobile phones had flip covers and the rest were without flip covers. The covered phones were further divided into interior and exterior regions to differentiate between the regions involved in direct and indirect exposure to the environment (Figure 1). As an extension to these, we also assessed the microbial load on human palm surfaces and the microbial transfer to human palms from phone surfaces (Figure 1). To check the microbial transfer to human palms from the phones, we sanitized the palms with $1.5 \mathrm{~mL}$ of Dettol Hand sanitizer (contact time of $15-20$ secs) before sample collection to ensure that the microbial population was entirely contributed by the mobile phones. Samples from mobile phones and palm surfaces were collected using sterile cotton swabs. Each swab was first 
moistened with sterile saline water and was rubbed over the surface of the cell phones and palms. The swab samples were also collected from the uncovered phones and the inner and outer surfaces of the phones with flip covers.

i)

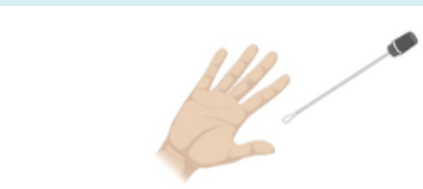

Swab rubbed over the palms on palm surfaces

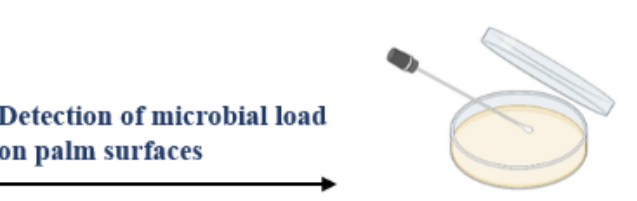

Streaking on agar plates

Detection of microbial load on mobile surfaces

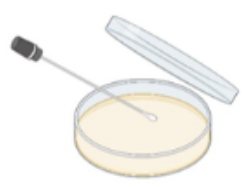

Swab rubbed over the phones

Streaking on agar plates

iii)

Microbial transfer from phones to palms

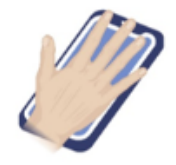

Palms rubbed over the phones
Swab rubbed over the palms from palms

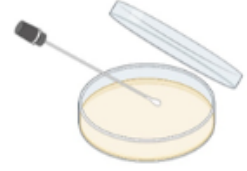

Streaking on agar plates

Figure 1: Schematic illustration for the detection of i) microbial load on palm surfaces, ii) microbial load on the mobile surfaces, and iii) microbial load on palm surfaces after being rubbed over mobile phones.

To get a hint of the antimicrobial efficacy of the sanitization process, samples were collected from palm surfaces exposed to the natural environment and also after the sanitization of the palms. The experiments were performed under the biological safety cabinet to ensure the elimination of external contamination. The sanitization efficiency was assessed using the following formula:

a) Sanitization efficiency against bacteria $(\%)=($ (total bacterial cfu before sanitization - total bacterial cfu after sanitization) $\div$ total bacterial cfu before sanitization) $\times$ $100 \%$

b) Sanitization efficiency against fungi (\%) = ((total fungal cfu before sanitization - total fungal cfu after sanitization) $\div$ total fungal cfu before sanitization) $\times 100 \%$

\section{Culturing of Microorganisms}

All the cotton swabs were streaked in three different media immediately under separated bacterial and fungal biological safety cabinets. The Mueller Hinton agar+ (MHA+) (supplemented with $100 \mu \mathrm{g} / \mathrm{mL}$ of Nystatin to avoid fungal contamination), Sabouraud Dextrose Agar+ (SDA+) (supplemented with $50 \mu \mathrm{g} / \mathrm{mL}$ of Chloramphenicol to avoid bacterial contamination), and Blood Agar (BA) plates were used for detecting the selective growth of bacteria, fungi, and fastidious organisms respectively. The MHA+ and the BA plates were incubated at $37^{\circ} \mathrm{C}$ for 24 hours, whereas the SDA+ plates were incubated at $37^{\circ} \mathrm{C}$ for 48 hours. After the incubation period, microbial colonies were counted from each of the plates. The process was carried out in triplicates to 
ensure reproducibility. For microbial identification, general protocols of Gram staining and Lacto-phenol cotton blue staining of pure cultures were performed for bacteria and fungi respectively $[15,16]$. The total microbial load (bacterial and fungal colony-forming units or $\mathrm{cfu}$ ) from each of the sections: i) Covered phones: exterior and interior regions, ii) uncovered phones, iii) palm surface rubbed against covered phones, and iv) palm surface rubbed against uncovered phones, were calculated, and the bacterial and fungal load were represented in percentage using the following formula:

a) Bacterial load (in \%) $=($ Bacterial cfu in $\mathrm{MHA}+$ and $\mathrm{BA}$ plates $\div$ total microbial cfu in MHA+, BA, and SDA+ plates) $\times 100 \%$

b) Fungal load (in \%) $=($ Fungal cfu in SDA + plates $\div$ total microbial cfu in MHA+, BA, and SDA+ plates) $\times 100 \%$

\section{Preparation of the Humectant-Hand-Sanitizer}

The alcohol-based sanitizer or HH-Sanitizer was prepared following our patent-pending formulation [17]. To prepare $100 \mathrm{~mL}$ of the solution, $85 \mathrm{~mL}$ of ethanol $(80 \% \mathrm{v} / \mathrm{v})$ was added into a glass container followed by the addition of $4.15 \mathrm{~mL}$ of hydrogen peroxide $(3 \% \mathrm{v} / \mathrm{v})$. A $1.5 \mathrm{~mL}$ of glycerol $(98 \% \mathrm{v} / \mathrm{v})$ was added further and the solution was gently mixed (either by shaking or using a paddle) for 2 hours. A 2 $\mathrm{mL}$ of chlorhexidine gluconate solution $(4 \% \mathrm{v} / \mathrm{v})$ was added, mixed gently and subsequently, $0.5 \mathrm{~mL}$ of a preservative, Germaben II $(0.7 \% \mathrm{v} / \mathrm{v})$ was added to the solution. Preautoclaved DI water was used to make up the volume of $100 \mathrm{~mL}$. The solution was quarantined for 72 hours before use which allowed the destruction of spores present in the alcohol or the containers used in the process. The $\mathrm{pH}$ of the solution would be 6.5-7.0. The manufacturing process was performed at room temperature. The composition used in the formulation was applied at a concentration that is below the permissible level (by the World Health Organization, and the Centers for Disease Control and Prevention) and therefore, reduces the chance of any toxic effects $[18,19]$.

\section{Antimicrobial efficiency of the HH-sanitizer}

The antimicrobial efficiency of the HH-sanitizer was assessed using the Zone of Inhibition (ZOI) and broth dilution methods against a group of bacteria and fungi often responsible for human-associated infections such as Enterobacter aerogenes, Escherichia coli, Klebsiella pneumoniae, Pseudomonas aeruginosa, Staphylococcus aureus, Enterococcus sp., Bacillus cereus, Listeria monocytogenes, Candida albicans, and Aspergillus sp. These isolates were selected for the study as they are often found as transient and resident flora on human skin surfaces [7]. Pure cultures of each bacterial and fungal isolates were grown in Mueller Hinton broth+ (MHB+) and Sabouraud dextrose broth $+(\mathrm{SDB}+)$ respectively. A $20 \mu \mathrm{L}$ of the test cultures maintained at $0.5 \mathrm{McF}$ arland standard were dropped on the MHA+ and SDA+ plates and spread by sterile L-shaped loops $[20,21]$. A $6 \mathrm{~mm}$ cork borer was used to bore holes on the $100 \mathrm{~mm}$ agar plates where $5 \%(\mathrm{v} / \mathrm{v})$ of the antimicrobial agent was added. The zone of inhibition was measured with the aid of a digital calliper following the CLSI guidelines [22]. To assess the minimum inhibitory concentration using the broth dilution method, six-twofold serial dilution of the agent $(5 \% \mathrm{v} / \mathrm{v})$ was prepared in $1 \mathrm{~mL}$ of $\mathrm{MHB}+$ and SDB+ media. A $100 \mu \mathrm{L}$ of the test cultures maintained at $0.5 \mathrm{McF}$ arland was added to each of the tubes. Turbidity was detected to verify bacterial and fungal growth. The plates and the tubes were incubated for $18 \mathrm{~h}$ for bacteria and $48 \mathrm{~h}$ for fungi at $37^{\circ} \mathrm{C}$. Pre-autoclaved DI water and Dettol sanitizer were used as controls. The experiments were performed in quadruplets to ensure reproducibility.

\section{Sanitization Efficiency of the HH-Sanitizer on Palm Surfaces}

The palm surfaces of the volunteers were sanitized using $1.5 \mathrm{~mL}$ of the $\mathrm{HH}$-sanitizer. The samples were collected before and after the palm surfaces were rubbed against the mobile phones. Here, we wanted to assess whether the sanitizer can eliminate microbes on palm surfaces exposed to the environment and after microbial transfer from phone surfaces at the same time or not. Sterile cotton swabs moistened with sterile saline water were rubbed over the palm surfaces and streaked in MHB+, BA, and SDA+ plates. The plates were incubated and the microbes were cultured as detailed earlier. The sanitization efficiency was calculated further.

\section{Statistical Analysis}

Statistical analysis of the data was carried out by OneWay ANOVA, Two-Way ANOVA (pairwise comparison was carried out by Bonferroni's posthoc test), Z-tests, and T-tests. All the statistical tests were carried out using GraphPad Prism 7.0, RStudio version 1.1.419 (using R version 3.4.3), graphs were plotted in GraphPad, RAW Graphs (DensityDesign Lab), and images were prepared using the Biorender application.

\section{Results and Discussion}

\section{Dettol Sanitizer Eliminates Bacteria with Higher Effectiveness than Fungi from Palm Surfaces}

The sanitizing process used to sanitize the palm surfaces of the volunteers gave a comparative idea as to what proportion, their palms are free of any microbial populations before sample collection. Palm surfaces exposed in the natural environment were found to be highly populated by bacterial species than fungal species. Precisely, the 
sanitization efficiency of Dettol sanitizer was observed to be 94.62\% against bacteria and 45.89\% against fungi (Figure 2 ). We inferred that the use of commercially available Dettol sanitizer was efficient in removing the bacterial population to a higher extent from the palm surfaces however failed in removing even $50 \%$ of the fungal population from the palm surfaces.

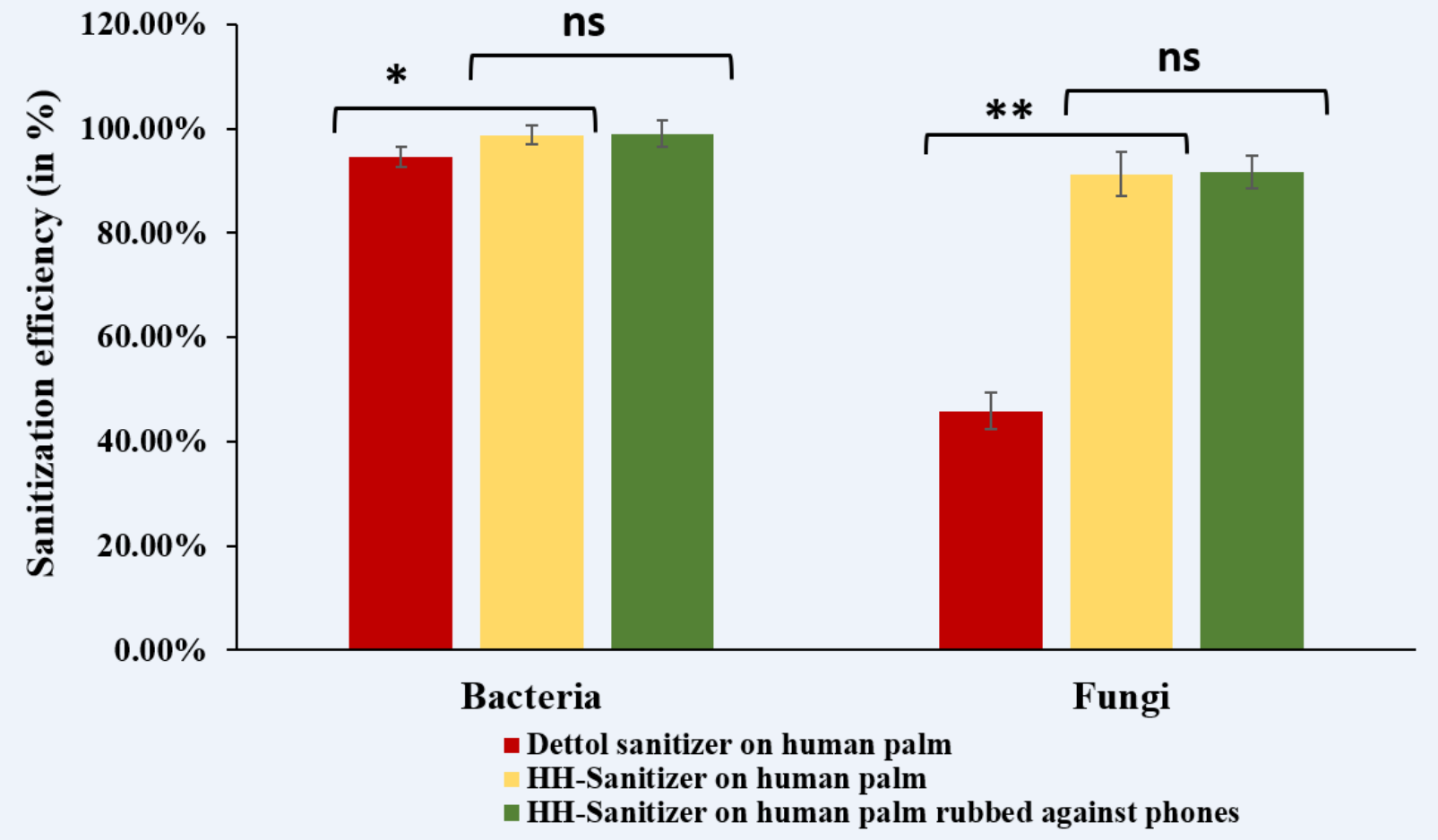

Figure 2: The sanitization efficiency of Dettol sanitizer on palm surfaces, HH-Sanitizer on palm surfaces, and HH-Sanitizer on palms after being rubbed over the phones. The sanitization efficiency of the HH-sanitizer on human palm exposed to the environment and after rubbing against phones was higher by 1.04 -fold $(\mathrm{p}<0.05)$ against bacteria, and 2 -fold $(\mathrm{p}<0.01)$ against fungi. A T-test was performed to assess the statistical significance.

\section{Phones with Flip Covers Have a Significantly Higher Bacterial Load Than Phones without Covers Whereas, More Fungal Species Adhered In Uncovered Mobile Phones}

The rate of bacterial load in the covered (96.71\%) and uncovered regions (65.72\%) of mobile phones was significantly different $(\mathrm{p}<0.0001)$ (Figure $3 \mathrm{a})$. Whereas, the fungal load observed from the covered (3.28\%) and uncovered phones $(34.27 \%)$ were much lower when compared to the bacterial colonies (Figure 3a). The bacterial and fungal groups showed opposite trends in terms of colony formation. The bacterial population (as per cfu) was found to be more than 13-fold higher in mobile phones with covers than the uncovered ones. The higher bacterial population on covered phones could be because the flip cover surface serves as a rough plateau-like platform such that bacteria can remain adhered to the surface by the excretion of extracellular polymeric substances (EPS) to enhance adhesion and biofilm formation. Surface roughness and surface topographical features physically affect bacterial adhesion on surfaces [23]. Previous studies revealed that adhesion forces increase with increasing surface roughness [23]. The bacterial adhesion was comparatively lower in uncovered phones as their surfaces were relatively smoother. The fungal population in the uncovered mobile phones was seen to be higher than that of the covered mobile phones. The primary reason could be that fungi adhere strongly to plastic-based surfaces by secreting an adhesive glue, galactosaminogalactan polymer [24]. Typically, mobile phone surfaces are mainly made up of either polycarbonate, polypropylene, polyurethane, or thermoplastic polyurethane. 

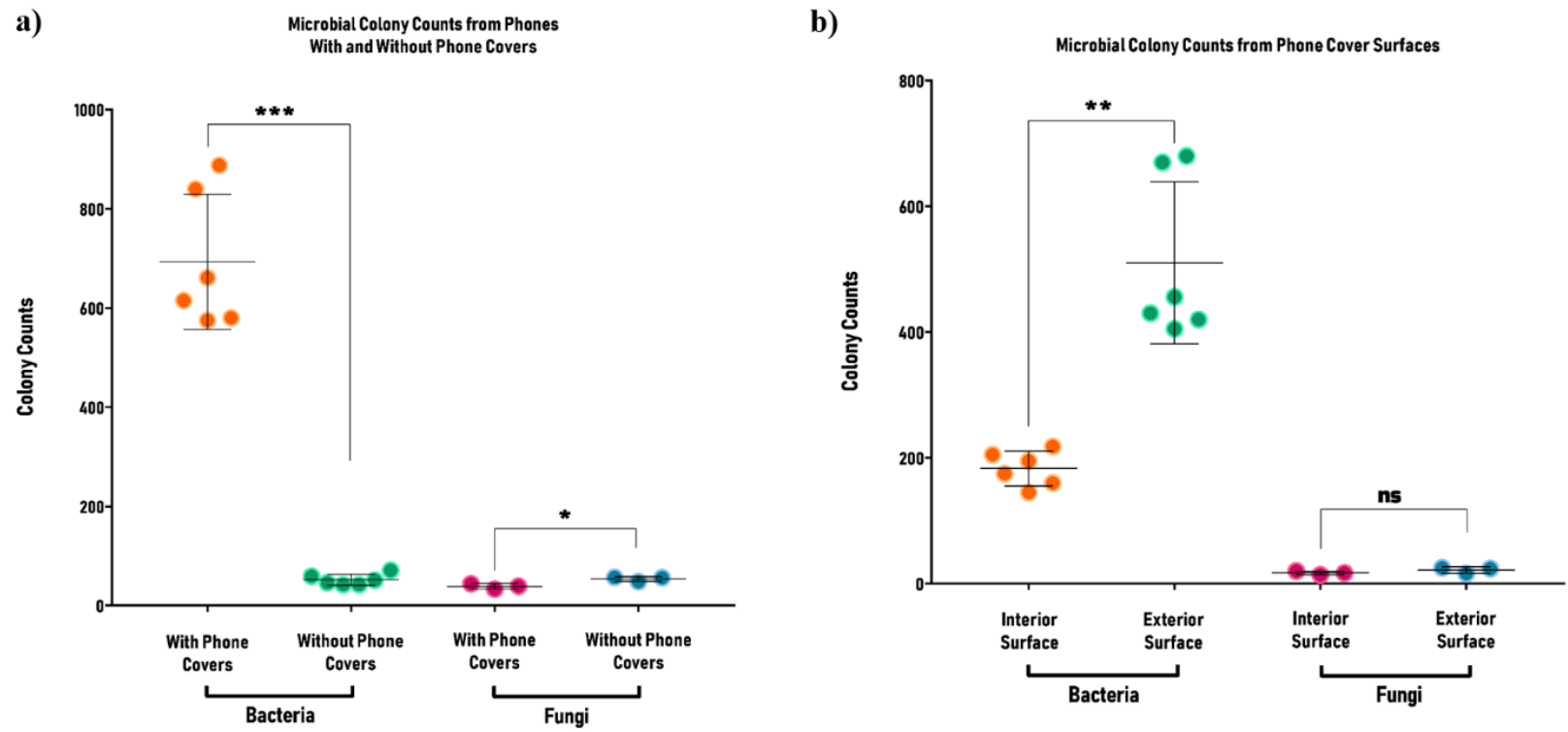

Figure 3: The total number of bacterial and fungal colonies obtained from a) covered phones and uncovered phones, and b) interior and exterior surfaces of phones with flip covers. Each symbol indicates one replicate ( $N=3$; $n=6$ experiments for bacteria and $n=3$ for fungi). Two-Way ANOVA and Bonferroni's posthoc test was used for comparison. Results indicate that bacterial and fungal groups show opposite trends in terms of colonies obtained from phone swabs. The number of bacterial colonies obtained was several times higher than that of fungal colonies for phones with covers ( $p<0.0001$ ), whereas the difference in colony number was non-significant in the case of phones without covers $(p=0.8023)$. Bacterial load was much higher on exterior surfaces of phone covers $(p<0.01)$, while the difference for fungi was non-significant $(p=0.234)$.

\section{Exterior Regions of Covered Phones Had a Significantly Higher Bacterial Load}

The rate of bacterial load in the exterior $(70.96 \%)$ and interior $(26.25 \%)$ regions of the covered mobile phones were significantly different $(\mathrm{p}<0.01)$ (Figure $3 \mathrm{~b})$. Whereas, the fungal load in the exterior (1.53\%) and interior $(1.24 \%)$ regions was found to be non-significant ( $\mathrm{p}>0.05$ ) (Figure $3 \mathrm{~b}$ ). The bacterial and fungal load was observed to be higher in the exterior regions of the flip cover than that of its interior region, which could be because the exterior region of the flip cover remains exposed to the natural environment compared to the interior region.

\section{Bacterial Transfer from Covered Phone Surfaces to Palm Surfaces was Higher, Whereas No Radical Change was Noted in Fungal Transfer}

The rate of bacterial load on the palm surfaces after rubbing the covered (97.61\%) and uncovered (65.21\%) regions of phones was statistically different $(\mathrm{p}<0.001)$ (Figure 4a). The bacterial population (as per cfu) was found to be more than 14.5-fold higher from the mobile phones with covers than the uncovered ones. The rate of fungal load obtained from the palm surfaces after rubbing the covered and uncovered phones were $2.38 \%$ and $34.78 \%$ respectively (Figure 4a). As the bacterial population was more in the covered region, it could be hypothesized that a higher bacterial load got transferred into the palm surface when rubbed against the covered region than that of the uncovered region. Similarly, a higher fungal load got transferred into the palm surface when rubbed against the uncovered region than that of the covered region. Another reason for the outcomes could be the presence of bacteria and fungi in the palm even after the sanitization process, as the sanitization efficacy of the Dettol sanitizer against bacteria and fungi was found to be $94.62 \%$ and $45.89 \%$ respectively which might have interfered in the true transfer of microbes from the phone surfaces to palms and hence, have increased the microbial population. 
a)

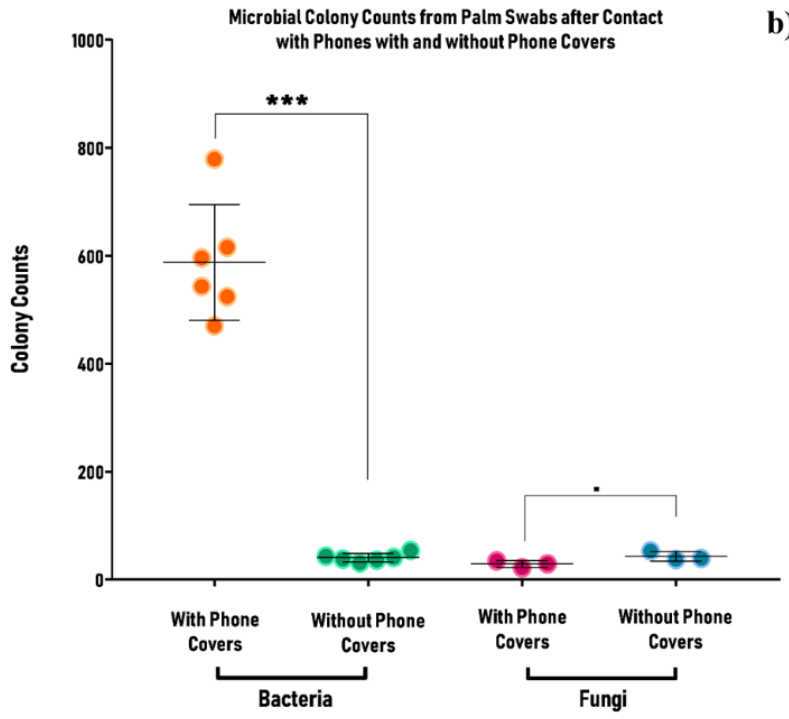

b)

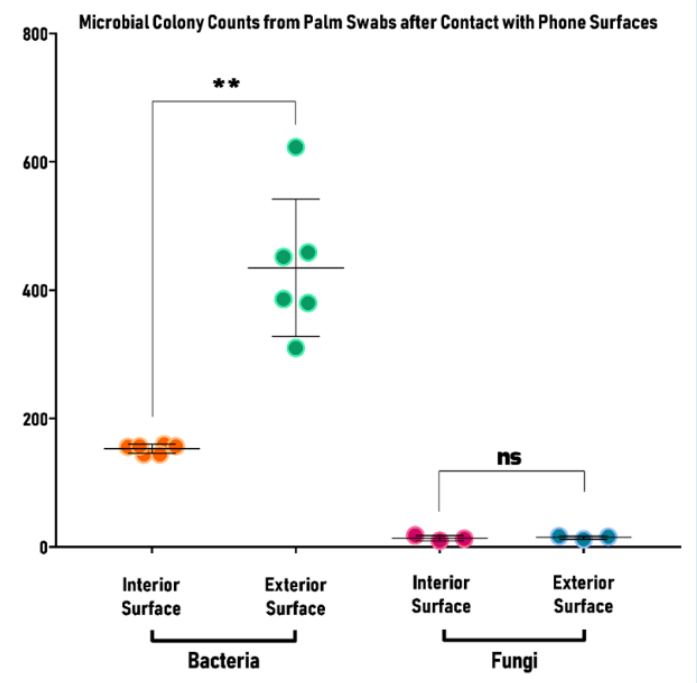

Figure 4: The total number of bacterial and fungal colonies obtained from a) palm swabs after contacting phones with and without phone covers, and b) palm swabs after contacting interior and exterior surfaces of the phones with flip covers. Each symbol indicates one replicate $(\mathrm{N}=3 ; \mathrm{n}=6$ experiments for bacteria and $\mathrm{n}=3$ for fungi). Two-Way ANOVA and Bonferroni's posthoc test was used for comparison. The number of bacterial colonies plated after contact with phones with covers was significantly higher than that obtained after contact with phones without covers ( $p<0.001)$, while for fungi, the number of colonies obtained from touching uncovered phones was marginally higher $(p=0.0846)$. The number of bacterial colonies plated after contact with exterior surfaces was significantly higher than that obtained after contact with interior surfaces $(\mathrm{p}<$ 0.01), while for fungi, the difference was non-significant.

\section{Bacterial Transfer From Exterior Regions of Covered Phones To The Palm Surfaces Were Significantly Higher}

The rate of bacterial load on the palm surfaces after rubbing the exterior $(71.75 \%)$ and interior $(25.86 \%)$ regions of the covered phones were statistically different $(\mathrm{p}<0.001)$ (Figure $4 \mathrm{~b}$ ). Whereas, the fungal load in the exterior $(1.24 \%$ ) and interior $(1.13 \%)$ regions was non-significant ( $p>0.05)$ (Figure $4 \mathrm{~b}$ ). As the bacterial and fungal population was higher in the exterior region of the covered phones in comparison to the interior surface, therefore a greater number of bacteria and fungi probably got transmitted into the palm surface when rubbed against the exterior region. However, the presence of bacteria and fungi even after the sanitization process could also interfere in the true assessment of microbial transfer.

\section{Humectant-Hand-Sanitizer was Effective Against Opportunistic Pathogens and Significantly Reduced Microbial Load from Palm Surfaces}

The antimicrobial tests of the HH-sanitizer depicted an excellent antibacterial and antifungal efficiency. Briefly, the mean ZOI demonstrated by the sanitizer against the Gramnegative (Enterobacter aerogenes, Escherichia coli, Klebsiella pneumoniae, Pseudomonas aeruginosa) Gram-positive (Staphylococcus aureus, Enterococcus sp., Bacillus cereus, Listeria monocytogenes), and fungal (Candida albicans, and Aspergillus sp.) isolates were $>17.5 \mathrm{~mm},>19 \mathrm{~mm}$, and $>15$ $\mathrm{mm}$ respectively which were higher than the ZOI-approved limit by the Clinical and Laboratory Standard Institute (CLSI) [22] (Table 1). Moreover, based on the ZOI test, HH-sanitizer was $\sim 2.5$-fold more efficient against the test organisms when compared to the Dettol sanitizer. The minimum inhibitory concentration of the sanitizer against the Gram-negative bacteria and fungal isolates was $0.62 \%(\mathrm{v} / \mathrm{v})$, and $0.31 \%$ (v/v) against the Gram-positive bacteria (Table 1). The MIC of the HH-sanitizer was 8-folds lower than that of the Dettolsanitizer when tested against the bacterial and fungal isolates. Interestingly, the Dettol sanitizer was comparatively effective against bacterial load present on the palm surfaces, however, failed to show efficiency against the test organisms. One of the reasons for this observation could probably be the induction of tolerance mechanism in microbes when they were exposed to the Dettol sanitizer for a longer duration (incubation for 24 $\mathrm{h}$ or $48 \mathrm{~h}$ ) [25]. Meanwhile, it was effective against bacterial load on palm surfaces as the samples were collected after an optimal period of 15-20 secs. Another reason could be the early evaporation of the active agents in the Dettol sanitizer. 


\begin{tabular}{|c|c|c|c|c|}
\hline \multirow{2}{*}{ Isolates } & \multicolumn{2}{|c|}{ Zone of inhibition (in mm) } & \multicolumn{2}{c|}{$\begin{array}{c}\text { Minimum inhibitory concentration } \\
\text { (in \% v) }\end{array}$} \\
\hline Enterobacter aerogenes & $<8.5$ & HH-sanitizer & Dettol sanitizer & HH-sanitizer \\
\hline Escherichia coli & $<8.5$ & $17.51 \pm 0.20$ & $>5 \%$ & $0.62 \%$ \\
\hline Klebsiella pneumoniae & $<8.5$ & $19.48 \pm 1.18$ & $>5 \%$ & $0.62 \%$ \\
\hline Pseudomonas aeruginosa & $<8.5$ & $17.27 \pm 3.11$ & $>5 \%$ & $0.62 \%$ \\
\hline Staphylococcus aureus & $<9$ & $19.43 \pm 0.83$ & $2.50 \%$ & $0.62 \%$ \\
\hline Enterococcus sp. & $<9$ & $16.67 \pm 1.38$ & $2.50 \%$ & $0.31 \%$ \\
\hline Bacillus cereus & $<9$ & $24.84 \pm 3.59$ & $2.50 \%$ & $0.31 \%$ \\
\hline Listeria monocytogenes & $<9$ & $16.54 \pm 1.18$ & $2.50 \%$ & $0.31 \%$ \\
\hline Candida albicans & $<7$ & $16.47 \pm 0.55$ & $>5 \%$ & $0.31 \%$ \\
\hline Aspergillus sp. & $<7$ & $13.69 \pm 0.79$ & $>5 \%$ & $0.62 \%$ \\
\hline
\end{tabular}

Table 1: Antimicrobial efficiency of the Dettol and HH-sanitizer against bacterial and fungal isolates.

A normal human skin harbors bacteria of around $10^{2}$ and $10^{6} \mathrm{CFU} / \mathrm{cm}^{2}$, thus the transfer of bacteria from the hands to food, objects, or people plays an important role in the spread of many communicable diseases [26]. The tested organisms are known opportunistic pathogens and responsible for several diseases and infections associated with humans. For instance, E. coli, E. aerogenes, and P. aeruginosa cause urinary tract infection (UTI), bacteremia, lower respiratory tract infection, endocarditis, intra-abdominal infections, soft tissue infections, gastrointestinal infections, etc. [2729]. L. monocytogenes causes listeriosis which can lead to miscarriage, and premature delivery [30]. S. aureus can cause diseases like bacteremia and infective endocarditis [31]. $B$. cereus causes food poisoning, fatal non-gastrointestinal tract infections, and eye infections [32,33]. Fungal isolates such as Candida sp. can cause thrush, vaginal yeast infections, itching, and rashes in the skin [34] whereas, Aspergillus sp. when inhaled can cause asthma, allergic alveolitis, pneumonia, neonatal infections, etc. [35].

Greenaway et al. recently reported that a $1.5 \mathrm{~mL}$ dose of ethanol or hand sanitizers with 15-20 seconds of contact time yielded the most acceptable properties with no extreme negative consequences on human skin [36]. The sanitization efficiency of the $\mathrm{HH}$-sanitizer ( $\mathrm{of} 1.5 \mathrm{~mL}$ ) on the palm surfaces was $98.78 \pm 1.82 \%$ and $91.27 \pm 4.24 \%$ against bacterial and fungal isolates respectively (Figure 2). No significant difference was noticed in the sanitization efficiency of the sanitizer on the palm surfaces vs when rubbed against the phones. This observation infers that the sanitizer is effective not only against the microbes on palm surfaces exposed to the environment but also against the additional microbial load from phones. Recent studies reported that the use of alcohol between $60-80 \%$ shows bactericidal activity by membrane damage, and inhibition or uncoupling of mRNA and protein synthesis through effects on ribosomes and RNA polymerase, or associated with protein denaturation $[37,38]$. At least $70 \%$ ethanol is reported to eliminate fungal spores by interacting with cellular membranes increasing membrane permeability and causing leakage of solutes and cell lysis [39]. Hydrogen peroxide used in the formulation is scientifically proven as a cleansing agent, disinfectant, and is known for bacteriostatic, and sporicidal activity [40]. Glycerol is used to soften, hydrate, and moisturize the skin [41], whereas Chlorhexidine Gluconate acts as a skin cleanser and is effective against facultative anaerobes, aerobes, enveloped viruses, yeast, and fungi $[40,42]$. Germaben II used in the formulation acts as an antimicrobial preservative system with broad-spectrum activity [43].

Jain et al. checked the antibacterial efficiency of commercially available hand sanitizers such as Dettol, Lifebuoy, PureHands, etc., and found their ZOI to be less than $10 \mathrm{~mm}$ [7]. In a similar study, a group tested around 20 different commercially available hand sanitizers including Fresh up, Germ X, Safeguard, etc., and found that most of them were ineffective against pathogenic bacteria [14]. When compared to the Dettol sanitizer and the existing literature on commercial hygiene products, the HH-sanitizer showed better performance against a wide range of opportunistic pathogens in vitro, and also against microbes from palms and phone surfaces thus warranting its potential application in public hygiene.

\section{Conclusion}

From this study, it could be inferred that fungal species adhere mostly to smooth phone surfaces that don't have flip covers whereas, the mobile phones that contain flip covers showed negligible fungal adherence but a higher bacterial 
population. Owing to the higher bacterial population in the covered mobile phones especially in the exterior regions, a higher bacterial load got transferred to the human palm surfaces. Hence, it is suggested not to use flip covers on mobile phones/laptops/tablets, etc. as this owes to several bacterial infections that might affect our surveillance against leading a normal and healthy life. Even though the fungal population in the uncovered cell phones was considerably higher than that of the covered regions, it is normally beneficial to use them, as the common infections we are prone to are caused more by bacteria than fungus. Commercially available Dettol sanitizer was effective against bacteria, however, failed to eliminate even $50 \%$ of fungal isolates from human palm surfaces. However, in vitro assessment showed the inefficiency of the Dettol sanitizer against opportunistic pathogens. On the other hand, the Humectant-Hand-Sanitizer showed excellent efficiency against opportunistic pathogens. Also, a $1.5 \mathrm{~mL}$ of the sanitizer was effective in reducing $>98.5 \%$ and $>91 \%$ of the bacterial and fungal load respectively from palm and phone surfaces. Thus, we propose the potential application of the HH-sanitizer as an alternative to other sanitizers to improve public hygiene.

\section{Practical Applications}

In a developing country like India with $17.7 \%$ of the World's population [44], 21\% of the communicable diseases are connected to the lack of proper hygiene practices [45]. Our study aims to spread awareness among the public regarding the existence of a wide range of microorganisms on electronic devices and their potential transfer to human hands. Thereby the use of such gadgets specifically with flip covers in places such as hospitals, cafeterias, laboratories, etc. should be restricted. Public health departments should take initiatives by advertising, hosting social and educational events to educate people about the issue. Proper disinfection of the devices every day, avoiding flip covers, sanitization of hands before every meal would reduce the chances of contact-based adherence of device-borne pathogens to human hands.

\section{Acknowledgement}

The authors would like to thank the Lucid-Laboratory Pvt Ltd, Hyderabad for their help in the antimicrobial studies.

\section{References}

1. (1996) Infectious diseases kill over 17 million people a year: WHO warns of global crisis.

2. Al Ghamdi A, Ashshi A, Faidah H, Shukri H, Abdelmal S (2011) Bacterial contamination of computer keyboards and mice, elevator buttons and shopping carts 5(23):
3998-4003.

3. Pratt RJ, Pellowe C, Loveday HP, Robinson N, Smith GW, et al. (2001) The epic project: developing national evidence-based guidelines for preventing healthcare associated infections. Phase I: Guidelines for preventing hospital-acquired infections. Department of Health (England). J Hosp Infect 47: 3-82.

4. Di Martino P (2018) Bacterial adherence: much more than a bond. AIMS Microbiol 4(3): 563-566.

5. Abrams A (2017) Your Cell Phone Is 10 Times Dirtier Than a Toilet Seat. Here's What to Do About It.

6. Tagoe DN, Gyande VK, Ansah EO (2011) Bacterial contamination of mobile phones: When your mobile phone could transmit more than just a call. 2(10): WMC002294.

7. Jain VM, Karibasappa GN, Dodamani AS, Prashanth VK, Mali GV (2016) Comparative assessment of antimicrobial efficacy of different hand sanitizers: An in vitro study. Dent Res J (Isfahan) 13(5): 424-431.

8. Wendt C (2001) Hand hygiene-comparison of international recommendations. J Hosp Infect 48: 23-28.

9. Larson EL, Hughes CA, Pyrek JD, Sparks SM, Cagatay EU, et al. (1998) Changes in bacterial flora associated with skin damage on hands of health care personnel. Am J Infect Control 26(5): 513-521.

10. Winnefeld M, Richard MA, Drancourt M, Grob JJ (2000) Skin tolerance and effectiveness of two hand decontamination procedures in everyday hospital use. Br J Dermatol 143(3): 546-550.

11. Hammond B, Ali Y, Fendler E, Dolan M, Donovan S (2000) Effect of hand sanitizer use on elementary school absenteeism. Am J Infect Control 28(5): 340-346.

12. Bloomfield SF, Aiello AE, Cookson B, O Boyle C, Larson RNEL (2007) The effectiveness of hand hygiene procedures in reducing the risks of infections in home and community settings including handwashing and alcohol-based hand sanitizers. American journal of infection control 35(10): 27-64.

13. Reynolds SA, Levy F, Walker ES (2006) Hand sanitizer alert. Emerg Infect Dis 12(3): 527-529.

14. Hayat A, Munnawar F (2016) Antibacterial effectiveness of commercially available hand sanitizers. International Journal of Biology and Biotechnology 13(3): 427-431. 
15. Coico R (2006) Gram Staining. Current Protocols in Microbiology 00(1): 3C.1-3C.2.

16. Leck A (1999) Preparation of lactophenol cotton blue slide mounts. Community Eye Health 12(30): 24.

17. Majumder S (2019) Humectant hand, and surface sanitizer and process of preparing the same in Patent Application Publication, India.

18. Rutala WA (2008) Guideline for Disinfection and Sterilization in Healthcare Facilities. Centers for Disease Control and Prevention.

19. (2006) WHO Guidelines on hand hygiene in health care, W.H. Organization. World Alliance for Patient Safety.

20. Samadi FM, Suhail S, Sonam M, Sharma N, Singh S, et al. (2019) Antifungal efficacy of herbs. J Oral Biol Craniofac Res 9(1): 28-32.

21. Majumder S, Jung D, Ronholm J, George S (2021) Prevalence and mechanisms of antibiotic resistance in Escherichia coli isolated from mastitic dairy cattle in Canada. BMC Microbiology 21(1): 222.

22. CLSI, (2017) Performance standards for antimicrobial susceptibility testing. CLSI document M100-S27, Wayne, PA: Clinical Laboratory Standards Institute (CLSI).

23. Songer JG, Post KW (2004) Veterinary microbiologyE-book: bacterial and fungal agents of animal disease. Elsevier Health Sciences.

24. Epstein L, Nicholson RL (2016) Adhesion and Adhesives of Fungi and Oomycetes. Chapter 2.

25. Ingram LO (1990) Ethanol tolerance in bacteria. Crit Rev Biotechnol 9(4): 305-319.

26. Kimura AC, Johnson K, Palumbo MS, Hopkins J, Boase JC (2004) Multistate shigellosis outbreak and commercially prepared food, United States. Emerg Infect Dis 10(6): 1147-1149.

27. Alanazi MQ, Alqahtani FY, Aleanizy FS (2018) An evaluation of $E$. coli in urinary tract infection in emergency department at KAMC in Riyadh, Saudi Arabia: retrospective study. Ann Clin Microbiol Antimicrob 17(1): 3-3.

28. Susan L Fraser (2019) Enterobacter Infections.

29. Moradali MF, Ghods S, Rehm BHA (2017) Pseudomonas aeruginosa Lifestyle: A Paradigm for Adaptation,
Survival, and Persistence. Front Cell Infect Microbiol $7(39)$.

30. Farber JM, Peterkin PI (1991) Listeria monocytogenes, a food-borne pathogen. Microbiological reviews 55(3): 476-511.

31. Petti CA, Fowler VG (2003) Staphylococcus aureus bacteremia and endocarditis. Infect Dis Clin North Am 21(2): 219-233.

32. Granum PE, Lund T (1997) Bacillus cereus and its food poisoning toxins. FEMS Microbiology Letters 157(2): 223-228.

33. Bottone EJ (2010) Bacillus cereus, a volatile human pathogen. Clin Microbiol Rev 23(2): 382-398.

34. Mayer FL, Wilson D, Hube B (2013) Candida albicans pathogenicity mechanisms. Virulence 4(2): 119-128.

35. Dagenais TRT, Keller NP (2009) Pathogenesis of Aspergillus fumigatus in Invasive Aspergillosis. Clin Microbiol Rev 22(3): 447-465.

36. Greenaway RE, Ormandy K, Fellows C, Hollowood T (2018) Impact of hand sanitizer format (gel/foam/ liquid) and dose amount on its sensory properties and acceptability for improving hand hygiene compliance. J Hosp Infect 100(2): 195-201.

37. Golin AP, Choi D, Ghahary A (2020) Hand sanitizers: A review of ingredients, mechanisms of action, modes of delivery, and efficacy against coronaviruses. Am J Infect Control 48(9): 1062-1067.

38. Fendler, E, Ali Y, Hammond BS, Lyons MK, Kelley MB, et al., (2002) The impact of alcohol hand sanitizer use on infection rates in an extended care facility. Am J Infect Control 30(4): 226-233.

39. Rogawansamy S, Gaskin S, Taylor M, Pisaniello D (2015) An evaluation of antifungal agents for the treatment of fungal contamination in indoor air environments. Int J Environ Res Public health 12(6): 6319-6332.

40. (2008) Guideline for Disinfection and Sterilization in Healthcare Facilities.

41. Fluhr JA, Bornkessel A, Berardesca E (2005) Glyceroljust a moisturizer? biological and biophysical effects. pp: 227-243.

42. Shino B, Peedikayil FC, Jaiprakash SR, Bijapur GA, Kottayi S, et al. (2016) Comparison of Antimicrobial Activity of 
Chlorhexidine, Coconut Oil, Probiotics, and Ketoconazole on Candida albicans Isolated in Children with Early Childhood Caries: An In Vitro Study. Scientifica pp: 7061587.

43. Berke P, Steinberg DC, Rosen WE (1982) Germaben II a complete preservative system in clear liquid form. pp: 89-93.

44. (2020) India population.

45. (2020) India's water and sanitation crisis. 